\title{
Control of Hopf Bifurcation and Chaos in a Delayed Lotka-Volterra Predator-Prey System with Time-Delayed Feedbacks
}

\author{
Huitao Zhao, ${ }^{1,2}$ Yaowei Sun, ${ }^{1}$ and Zhen Wang ${ }^{3}$ \\ ${ }^{1}$ Department of Mathematics and Information Science, Zhoukou Normal University, Zhoukou, Henan 466001, China \\ ${ }^{2}$ Department of Applied Mathematics, Kunming University of Science and Technology, Kunming, Yunnan 650093, China \\ ${ }^{3}$ Institute of Information and System Computation Science, Beifang University of Nationalities, Yinchuan, Ningxia 750021, China \\ Correspondence should be addressed to Huitao Zhao; taohuiz@sohu.com
}

Received 10 March 2014; Accepted 19 May 2014; Published 4 June 2014

Academic Editor: Imran Naeem

Copyright (C) 2014 Huitao Zhao et al. This is an open access article distributed under the Creative Commons Attribution License, which permits unrestricted use, distribution, and reproduction in any medium, provided the original work is properly cited.

\begin{abstract}
A delayed Lotka-Volterra predator-prey system with time delayed feedback is studied by using the theory of functional differential equation and Hassard's method. By choosing appropriate control parameter, we investigate the existence of Hopf bifurcation. An explicit algorithm is given to determine the directions and stabilities of the bifurcating periodic solutions. We find that these control laws can be applied to control Hopf bifurcation and chaotic attractor. Finally, some numerical simulations are given to illustrate the effectiveness of the results found.
\end{abstract}

\section{Introduction}

Lotka-Volterra system is one of the most classical and important systems in the field of mathematical biology. Since the word of Volterra, there have been extensively detailed investigations on Lotka-Volterra system including stability, attractivity, persistence, periodic oscillation, bifurcation and chaos (see [1-6] and the references therein). In particular, the properties of periodic solutions arising from the Hopf bifurcation are of great interest [7-10]. But the study on chaos control of Lotka-Volterra system is scarce.

Reference [3] and the references therein proposed that, for a two-species competition system with delays

$$
\dot{u}_{i}(t)=u_{i}(t)\left[r_{i}+\sum_{j=1}^{n} a_{i j} u_{j}\left(t-\tau_{i j}\right)\right], \quad i=1,2,
$$

when $\tau_{i j}$ is big enough, the chaotic behavior may occur.
For example, Yan and Zhang [9] investigated the following delayed prey-predator system with a single delay:

$$
\begin{aligned}
& \dot{x}(t)=x(t)\left[r_{1}-a_{11} x(t-\tau)-a_{12} y(t-\tau)\right], \\
& \dot{y}(t)=y(t)\left[-r_{2}+a_{21} x(t-\tau)-a_{22} y(t-\tau)\right],
\end{aligned}
$$

where $r_{1}, r_{2}, a_{11}, a_{12}, a_{21}$, and $a_{22}$ are all positive constants. The delay $\tau \geq 0$ denotes the gestation period of the predator. Their results show that, taking $\tau$ as the bifurcation parameter, when $\tau$ passes through a certain critical value, the positive equilibrium loses its stability and Hopf bifurcation takes place. Furthermore, when $\tau$ takes a sequence of critical values containing the above critical value, the positive equilibrium of system (2) will undergo a Hopf bifurcation. With the further increase of the delay, the system will show the chaotic phenomenon (see Figure 1).

In the sense of biology, chaotic behavior sometimes is to the disadvantage of virtuous cycle and develop of the ecosystem, so we want to control this chaos phenomenon and 

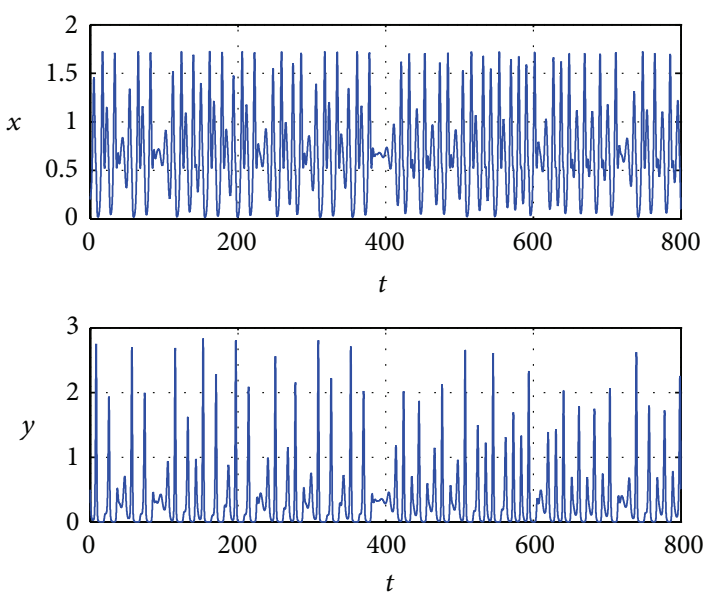

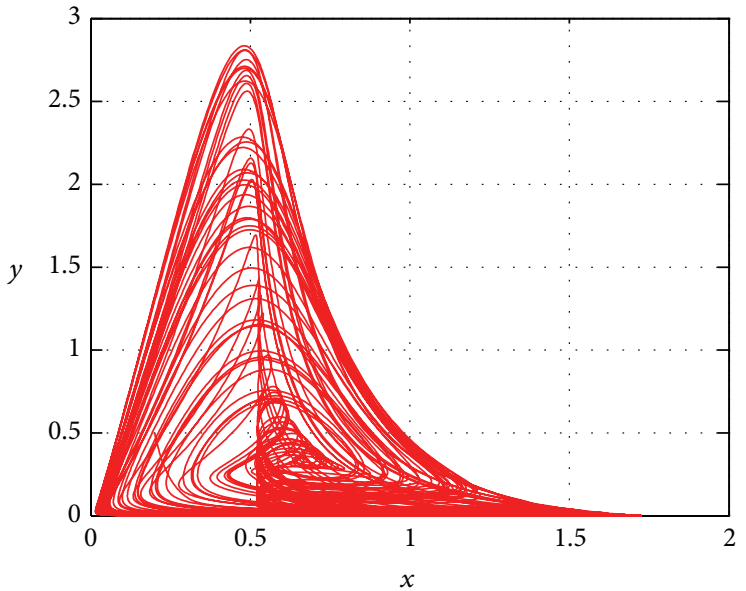

FIGURE 1: Waveform plot and phase plot of system (2) with $\tau=1.4$.

create periodic orbits. So far, many researchers have proposed chaos control schemes in recent years [11-16]. For example, Song and Wei in [17] investigated the chaos phenomena of Chen's system using the method of delayed feedback control. Their results show that, when the controlling parameter $K$ to be some value, taking the delay $\tau$ as the bifurcation parameter, when $\tau$ passes through a certain critical value, the stability of the equilibrium will be changed from unstable to stable, chaos vanishes, and a periodic solution emerges.

To the end of controlling chaos in system (2), stimulating by the works of above, we add some delayed feedback terms to system (2), that is, the following delayed feedback control system:

$$
\begin{aligned}
\dot{x}(t)= & x(t)\left[r_{1}-a_{11} x(t-\tau)-a_{12} y(t-\tau)\right] \\
& +k_{1}(x(t)-x(t-\tau)), \\
\dot{y}(t)= & y(t)\left[-r_{2}+a_{21} x(t-\tau)-a_{22} y(t-\tau)\right] \\
& +k_{2}(y(t)-y(t-\tau)),
\end{aligned}
$$

where $k_{i}(i=1,2)$ denote the capture coefficient when $k_{i}<0$ (or release coefficient when $k_{i}>0$ ). By choosing $\tau$ and $k_{i}$ as bifurcation parameter, we get the conditions under which Hopf bifurcation occurs. And then, we derive the explicit algorithm for determining the direction of the Hopf bifurcation and stability of the bifurcating periodic solutions. At last we will give some example showing that when $k_{i}$ is fixed, with $\tau$ increasing, the stability of the positive equilibrium will be changed, chaos vanishes, a periodic solution occurs.

This paper is organized as follows. In Section 2, we first focus on the stability and Hopf bifurcation of the positive equilibrium. In Section 3, we derive the direction and stability of Hopf bifurcation by using normal form and central manifold theory. Finally in Section 4, numerical simulations are performed to support the stability results.

\section{Stability and Hopf Bifurcation Analysis with Delayed Feedback Control}

In this section, by analyzing the characteristic equation of the linearized system of system (3) at the positive equilibrium, we investigate the stability of the positive equilibrium and the existence of the local Hopf bifurcations occurring at the positive equilibrium. To guarantee that system (3) has always a positive equilibrium, throughout this section, we assume that the coefficients of system (3) satisfy the following condition:

(H1) $a_{21} r_{1}-a_{11} r_{2}>0$.

Clearly, under the hypothesis (H1), system (3) has a unique positive equilibrium $E_{*}\left(x^{*}, y^{*}\right)$, where

$$
x^{*}=\frac{a_{22} r_{1}+a_{12} r_{2}}{a_{11} a_{22}+a_{12} a_{21}}, \quad y^{*}=\frac{a_{21} r_{1}-a_{11} r_{2}}{a_{11} a_{22}+a_{12} a_{21}} .
$$

Let $u_{1}(t)=x(t)-x^{*}, u_{2}(t)=y(t)-y^{*}$; then system (3) can be rewritten as the following equivalent system:

$$
\begin{aligned}
\dot{u}_{1}(t)= & \left(u_{1}(t)+x^{*}\right)\left[-a_{11} u_{1}(t-\tau)-a_{12} u_{2}(t-\tau)\right] \\
& +k_{1}\left(u_{1}(t)-u_{1}(t-\tau)\right), \\
\dot{u}_{2}(t)= & \left(u_{2}(t)+y^{*}\right)\left[a_{21} u_{1}(t-\tau)-a_{22} u_{2}(t-\tau)\right] \\
& +k_{2}\left(u_{2}(t)-u_{2}(t-\tau)\right) .
\end{aligned}
$$

Thus, the positive equilibrium $E_{*}\left(x^{*}, y^{*}\right)$ of system (3) is transformed into the equilibrium $(0,0)$ of system $(5)$. 
Linearizing system $(5)$ at the equilibrium $(0,0)$ yields the following linear system:

$$
\begin{aligned}
\dot{u}_{1}(t)= & -a_{11} x^{*} u_{1}(t-\tau)-a_{12} x^{*} u_{2}(t-\tau) \\
& +k_{1}\left(u_{1}(t)-u_{1}(t-\tau)\right), \\
\dot{u}_{2}(t)= & a_{21} y^{*} u_{1}(t-\tau)-a_{22} y^{*} u_{2}(t-\tau) \\
& +k_{2}\left(u_{2}(t)-u_{2}(t-\tau)\right) .
\end{aligned}
$$

The characteristic equation of system (6) is

$$
\lambda^{2}+a_{1} \lambda+a_{2}+a_{3} e^{-\lambda \tau}+a_{4} \lambda e^{-\lambda \tau}+a_{5} e^{-2 \lambda \tau}=0,
$$

where $a_{1}=-\left(k_{1}+k_{2}\right), a_{2}=k_{1} k_{2}, a_{3}=-\left(k_{1} a_{22} y^{*}+k_{2} a_{11} x^{*}+\right.$ $\left.2 k_{1} k_{2}\right), a_{4}=a_{11} x^{*}+a_{22} y^{*}+k_{1}+k_{2}, a_{5}=a_{11} a_{22} x^{*} y^{*}+$ $a_{12} a_{21} x^{*} y^{*}+k_{1} a_{22} y^{*}+k_{2} a_{11} x^{*}+k_{1} k_{2}$. Multiplying $e^{\lambda \tau}$ by both sides of (8), we have

$$
\left(\lambda^{2}+a_{1} \lambda+a_{2}\right) e^{\lambda \tau}+a_{3}+a_{4} \lambda+a_{5} e^{-\lambda \tau}=0
$$

Thus, $i \omega(\omega>0)$ is a root of (8) if and only if $\omega$ satisfies the following equation:

$$
\begin{aligned}
& \left(-\omega^{2}+a_{1} i \omega+a_{2}\right)(\cos \omega \tau+i \sin \omega \tau)+a_{3}+a_{4} i \omega \\
& +a_{5}(\cos \omega \tau-i \sin \omega \tau)=0 .
\end{aligned}
$$

Separating the real and imaginary parts, we have

$$
\begin{aligned}
& \left(\omega^{2}-a_{2}-a_{5}\right) \cos \omega \tau+a_{1} \omega \sin \omega \tau=a_{3}, \\
& \left(\omega^{2}-a_{2}+a_{5}\right) \sin \omega \tau-a_{1} \omega \cos \omega \tau=a_{4} \omega .
\end{aligned}
$$

It follows that

$$
\begin{aligned}
& \sin \omega \tau=\frac{d_{3} \omega^{3}+d_{4} \omega}{\omega^{4}+d_{1} \omega^{2}+d_{2}}, \\
& \cos \omega \tau=\frac{d_{5} \omega^{2}+d_{6}}{\omega^{4}+d_{1} \omega^{2}+d_{2}},
\end{aligned}
$$

where $d_{1}=a_{1}^{2}-2 a_{2}, d_{2}=a_{2}^{2}-a_{5}^{2}, d_{3}=a_{4}, d_{4}=a_{1} a_{3}-a_{2} a_{4}-$ $a_{4} a_{5}, d_{5}=a_{3}-a_{1} a_{4}, d_{6}=a_{3} a_{5}-a_{2} a_{3}$. From $\sin ^{2} \omega \tau+\cos ^{2} \omega \tau=$ 1 , we have

$$
\omega^{8}+e_{1} \omega^{6}+e_{2} \omega^{4}+e_{3} \omega^{2}+e_{4}=0,
$$

where $e_{1}=2 d_{1}-d_{3}^{2}, e_{2}=2 d_{2}+d_{1}^{2}-d_{5}^{2}-2 d_{3} d_{4}, e_{3}=2 d_{1} d_{2}-$ $2 d_{5} d_{6}-d_{4}^{2}, e_{4}=d_{2}^{2}-d_{6}^{2}$.

Denote $z=\omega^{2}$; (12) becomes

$$
z^{4}+e_{1} z^{3}+e_{2} z^{2}+e_{3} z+e_{4}=0
$$

Let

$$
G(z)=z^{4}+e_{1} z^{3}+e_{2} z^{2}+e_{3} z+e_{4}
$$

Since $\lim _{z \rightarrow \infty} G(z)=+\infty$, we conclude that if $e_{4}<0$, then (13) has at least one positive root. If all the parameters of system (5) are given, it is easy to get the roots of (13) by using a computer. Suppose the following.

(H2) Equation (13) has at least one positive real root. Without loss of generality, we assume that (14) has four positive roots, defined by $z_{1}, z_{2}, z_{3}, z_{4}$, respectively. Then (12) has four positive roots

$$
\omega_{1}=\sqrt{z_{1}}, \quad \omega_{2}=\sqrt{z_{2}}, \quad \omega_{3}=\sqrt{z_{3}}, \quad \omega_{4}=\sqrt{z_{4}} .
$$

From (11), we have

$$
\cos \omega_{k} \tau=\frac{d_{5} \omega_{k}^{2}+d_{6}}{\omega_{k}^{4}+d_{1} \omega_{k}^{2}+d_{2}}
$$

If we denote

$$
\begin{array}{r}
\tau_{k}^{(j)}=\frac{1}{\omega_{k}}\left\{\arccos \left(\frac{d_{5} \omega_{k}^{2}+d_{6}}{\omega_{k}^{4}+d_{1} \omega_{k}^{2}+d_{2}}\right)+2 j \pi\right\}, \\
j=0,1,2, \ldots,
\end{array}
$$

where $k=1, \ldots, 4 ; j=0,1, \ldots$, then $\pm i \omega_{k}$ is a pair of purely imaginary roots of (8) with $\tau=\tau_{k}^{(j)}$.

Define

$$
\tau_{0}=\tau_{k_{0}}^{(0)}=\min _{k=1, \ldots, 4}\left\{\tau_{k}^{(0)}\right\}, \quad \omega_{0}=\omega_{k_{0}} .
$$

Let $\lambda(\tau)=\alpha(\tau)+i \omega(\tau)$ be the root of (8) near $\tau=\tau_{k}^{(j)}$ satisfying $\alpha\left(\tau_{k}^{(j)}\right)=0, \omega\left(\tau_{k}^{(j)}\right)=\omega_{k}$. Substituting $\lambda(\tau)$ into (8) and taking the derivative with respect to $\tau$, we have

$$
\begin{aligned}
& \left(2 \lambda+a_{1}\right) e^{\lambda \tau} \frac{d \lambda}{d \tau} \\
& +\left(\lambda^{2}+a_{1} \lambda+a_{2}\right) e^{\lambda \tau}\left(\lambda+\tau \frac{d \lambda}{d \tau}\right) \\
& +a_{4} \frac{d \lambda}{d \tau}-a_{5} e^{-\lambda \tau}\left(\lambda+\tau \frac{d \lambda}{d \tau}\right)=0 .
\end{aligned}
$$

It follows that

$$
\frac{d \lambda}{d \tau}=\frac{a_{5} \lambda e^{-\lambda \tau}-\left(\lambda^{2}+a_{1} \lambda+a_{2}\right) \lambda e^{\lambda \tau}}{\left(2 \lambda+a_{1}\right) e^{\lambda \tau}+\left(\lambda^{2}+a_{1} \lambda+a_{2}\right) \tau e^{\lambda \tau}-a_{5} \tau e^{-\lambda \tau}+a_{4}} .
$$

Then

$$
\left[\frac{d \lambda}{d \tau}\right]^{-1}=\frac{\left(2 \lambda+a_{1}\right) e^{\lambda \tau}+a_{4}}{a_{5} \lambda e^{-\lambda \tau}-\left(\lambda^{2}+a_{1} \lambda+a_{2}\right) \lambda e^{\lambda \tau}}-\frac{\tau}{\lambda} .
$$


Through tedious calculating, we can get

$$
\left[\frac{d \lambda}{d \tau}\right]_{\tau=\tau_{k}^{(j)}}^{-1}=\frac{E+i F}{C+i D}-\frac{\tau_{k}^{(j)}}{i \omega_{k}}
$$

where

$$
\begin{aligned}
C= & a_{5} \omega_{k} \sin \omega_{k} \tau_{k}^{(j)}-\left(\omega_{k}^{2}-a_{2}\right) \omega_{k} \sin \omega_{k} \tau_{k}^{(j)} \\
& +a_{1} \omega_{k}^{2} \cos \omega_{k} \tau_{k}^{(j)}, D \\
= & a_{5} \omega_{k} \cos \omega_{k} \tau_{k}^{(j)}+\left(\omega_{k}^{2}-a_{2}\right) \omega_{k} \cos \omega_{k} \tau_{k}^{(j)} \\
& +a_{1} \omega_{k}^{2} \sin \omega_{k} \tau_{k}^{(j)}, E \\
= & a_{1} \cos \omega_{k} \tau_{k}^{(j)}-2 \omega_{k} \sin \omega_{k} \tau_{k}^{(j)}+a_{4}, F \\
= & a_{1} \sin \omega_{k} \tau_{k}^{(j)}+2 \omega_{k} \cos \omega_{k} \tau_{k}^{(j)} .
\end{aligned}
$$

Denote $Q=C^{2}+D^{2}$; then $Q>0$, and we have

$$
Q \operatorname{Re}\left[\frac{d \lambda}{d \tau}\right]_{\tau=\tau_{k}^{(j)}}^{-1}=E C+D F
$$

and note that

$$
\begin{aligned}
& \operatorname{sign}\left\{\operatorname{Re}\left[\frac{d \lambda}{d \tau}\right]_{\tau=\tau_{k}^{(j)}}\right\} \\
& =\operatorname{sign}\left\{\operatorname{Re}\left[\frac{d \lambda}{d \tau}\right]_{\tau=\tau_{k}^{(j)}}^{-1}\right\}=\operatorname{sign}\left\{Q \operatorname{Re}\left[\frac{d \lambda}{d \tau}\right]_{\tau=\tau_{k}^{(j)}}^{-1}\right\} .
\end{aligned}
$$

In order to get the main results, it is necessary to make the following assumption.

(H3) $E C+D F \neq 0$. Then, if (H3) holds, the transversality conditions $\operatorname{Re}[d \lambda / d \tau]_{\tau=\tau_{0}} \neq 0$ hold.

Note that when $\tau=0$, (8) becomes

$$
\lambda^{2}+\left(a_{1}+a_{4}\right) \lambda+a_{2}+a_{3}+a_{5}=0 .
$$

From [9], we know that all the roots of (26) have negative real parts; hence, the positive equilibrium $E_{*}$ is locally asymptotically stable for $\tau=0$. Then, we can employ a result of Ruan and Wei [18] to analyze (8). For the convenience of the reader, we state it as follows.

Lemma 1. Consider the exponential polynomial

$$
\begin{aligned}
P(\lambda, & \left.e^{-\lambda \tau_{1}}, \ldots, e^{-\lambda \tau_{m}}\right) \\
= & \lambda^{n}+p_{1}^{(0)} \lambda^{n-1}+\cdots+p_{n-1}^{(0)} \lambda+p_{n}^{(0)} \\
& +\left[p_{1}^{(1)} \lambda^{n-1}+\cdots+p_{n-1}^{(1)} \lambda+p_{n}^{(1)}\right] e^{-\lambda \tau_{1}} \\
& +\cdots+\left[p_{1}^{(m)} \lambda^{n-1}+\cdots+p_{n-1}^{(m)} \lambda+p_{n}^{(m)}\right] e^{-\lambda \tau_{m}},
\end{aligned}
$$

where $\tau_{i} \geqslant 0(i=1,2, \ldots, m)$ and $p_{j}^{(i)}(i=0,1, \ldots, m ; j=$ $1,2, \ldots, n)$ are constants. As $\left(\tau_{1}, \tau_{2}, \ldots, \tau_{m}\right)$ vary, the sum of the order of the zeroes of $P\left(\lambda, e^{-\lambda \tau_{1}}, \ldots, e^{-\lambda \tau_{m}}\right)$ on the open right half plane can change only if a zero appears on or crosses the imaginary axis.

From Lemma 1 and the above assumption, we can obtain the following theorem.

Theorem 2. Suppose that (H2) and (H3) hold; then the following results hold true.

(i) The positive equilibrium $E_{*}\left(x^{*}, y^{*}\right)$ of system (3) is asymptotically stable for $\tau \in\left[0, \tau_{0}\right)$;

(ii) system (3) exhibits Hopf bifurcation at the positive equilibrium for $\tau=\tau_{0}$.

\section{Direction and Stability of the Hopf Bifurcation}

In this section, we obtain the conditions under which a family periodic solutions bifurcate form the steady state at the critical value of $\tau$. As pointed out by Hale and Verduyn Lunel [19] and Hassard et al. [20], it is interesting to determine the direction, stability, and period of these periodic solutions bifurcating from the steady state. Following the ideal of [20], we derive the explicit formulae for determining the properties of the Hopf bifurcation at the critical value of $\tau$ using the normal form and the center manifold theory.

For the sake of simplicity of notation, we denote the critical values $\tau_{j}^{(k)}$ as $\tau_{k}$, and when $\tau=\tau_{k}$, we denote the pair of purely imaginary roots of (8) as $\pm i \omega_{k}$. Let $\mu=\tau-\tau_{k}$; then $\mu=0$ is the Hopf bifurcation value of system (5). In the following, we consider the equivalent system (6). Let $t=\tau t$; then the system (5) can be rewritten as a functional differential equation in $\mathbb{C}\left([-1,0], \mathbb{R}^{2}\right)$ :

$$
\dot{x}(t)=L_{\mu}\left(x_{t}\right)+f\left(\mu, x_{t}\right),
$$

where $x=\left(u_{1}, u_{2}\right)^{T}$, For $\phi=\left(\phi_{1}, \phi_{2}\right)^{T} \in \mathbb{C}\left([-1,0], \mathbb{R}^{2}\right)$,

$$
\begin{aligned}
L_{\mu}(\phi)= & \left(\tau_{k}+\mu\right)\left[\begin{array}{cc}
k_{1} & 0 \\
0 & k_{2}
\end{array}\right]\left[\begin{array}{l}
\phi_{1}(0) \\
\phi_{2}(0)
\end{array}\right] \\
& +\left(\tau_{k}+\mu\right)\left[\begin{array}{cc}
-a_{11} x^{*}-k_{1} & -a_{12} x^{*} \\
a_{21} y^{*} & -a_{22} y^{*}-k_{2}
\end{array}\right]\left[\begin{array}{l}
\phi_{1}(-1) \\
\phi_{2}(-1)
\end{array}\right], \\
f(\mu, \phi) & =\left(\tau_{k}+\mu\right)\left[\begin{array}{c}
-a_{11} \phi_{1}(0) \phi_{1}(-1)-a_{12} \phi_{1}(0) \phi_{2}(-1) \\
a_{21} \phi_{2}(0) \phi_{1}(-1)-a_{22} \phi_{2}(0) \phi_{2}(-1)
\end{array}\right] .
\end{aligned}
$$

Obviously, $L(\mu)$ is a continuous linear function mapping $\mathbb{C}\left([-1,0], \mathbb{R}^{2}\right)$ into $\mathbb{R}^{2}$. By the Riesz representation theorem, 
there exists a $2 \times 2$ matrix function $\eta(\theta, \mu)(-1 \leqslant \theta \leqslant 0)$, whose elements are of bounded variation such that

$$
L_{\mu} \phi=\int_{-1}^{0} d \eta(\theta, 0) \phi(\theta), \quad \text { for } \phi \in \mathbb{C}\left([-1,0], \mathbb{R}^{2}\right) \text {. }
$$

In fact, we can choose

$$
\begin{aligned}
\eta(\theta, \mu)= & \left(\tau_{k}+\mu\right)\left[\begin{array}{cc}
k_{1} & 0 \\
0 & k_{2}
\end{array}\right] \delta(\theta) \\
& -\left(\tau_{k}+\mu\right)\left[\begin{array}{cc}
-a_{11} x^{*}-k_{1} & -a_{12} x^{*} \\
a_{21} y^{*} & -a_{22} y^{*}-k_{2}
\end{array}\right] \delta(\theta+1),
\end{aligned}
$$

where $\delta$ denotes Dirac-delta function. For $\phi \in \mathbb{C}\left([-1,0], \mathbb{R}^{2}\right)$, define

$$
\begin{aligned}
& A(\mu) \phi= \begin{cases}\frac{d \phi(\theta)}{d \theta}, & \theta \in[-1,0), \\
\int_{-1}^{0} d \eta(s, \mu) \phi(s), & \theta=0,\end{cases} \\
& R(\mu) \phi= \begin{cases}0, & \theta \in[-1,0), \\
f(\mu, \phi), & \theta=0 .\end{cases}
\end{aligned}
$$

$$
\tau_{k}\left[\begin{array}{cc}
i \omega_{k}-k_{1}+\left(a_{11} x^{*}+k_{1}\right) e^{-i \omega_{k} \tau_{k}} & a_{12} x^{*} e^{-i \omega_{k} \tau_{k}} \\
-a_{21} y^{*} e^{-i \omega_{k} \tau_{k}} & i \omega_{k}-k_{2}+\left(a_{22} y^{*}+k_{2}\right) e^{-i \omega_{k} \tau_{k}}
\end{array}\right] q(0)=\left[\begin{array}{l}
0 \\
0
\end{array}\right] .
$$

Thus, we can easily obtain $\alpha=-a_{21} y^{*} e^{-i \omega_{k} \tau_{k}} /\left(i \omega_{k}-k_{2}+\right.$ $\left.\left(a_{22} y^{*}+k_{2}\right) e^{-i \omega_{k} \tau_{k}}\right), q(0)=(1, \alpha)^{T}$.

Similarly, let $q^{*}(s)=D\left(1, \alpha^{*}\right) e^{i \omega_{k} \tau_{k} s}$ be the eigenvector of $A^{*}$ corresponding to $-i \omega_{k} \tau_{k}$. By the definition of $A^{*}$, we can compute $\alpha^{*}=a_{12} x^{*} e^{i \omega_{k} \tau_{k}} /\left(i \omega_{k}+k_{2}-\left(a_{22} y^{*}+k_{2}\right) e^{i \omega_{k} \tau_{k}}\right)$.

In order to assure $\left\langle q^{*}(s), q(\theta)\right\rangle=1$, we need to determine the value of $D$. From (36), we have

$$
\begin{aligned}
& \left\langle q^{*}(s), q(\theta)\right\rangle \\
& =\bar{D}\left(1, \bar{\alpha}^{*}\right)(1, \alpha)^{T} \\
& \quad-\int_{-1}^{0} \int_{\xi=0}^{\theta} \bar{D}\left(1, \bar{\alpha}^{*}\right) e^{-i \omega_{k} \tau_{k}(\xi-\theta)} d \eta(\theta)(1, \alpha)^{T} e^{i \omega_{k} \tau_{k} \xi} d \xi \\
& =\bar{D}\left\{1+\alpha \bar{\alpha}^{*}+\tau_{k}\left(-a_{11} x^{*}-k_{1}+a_{21} y^{*} \bar{\alpha}^{*}-a_{12} x^{*} \alpha\right.\right. \\
& \left.\left.\quad-\left(a_{22} y^{*}+k_{2}\right) \alpha \bar{\alpha}^{*}\right) e^{-i \omega_{k} \tau_{k}}\right\} .
\end{aligned}
$$

Thus, we can choose

$$
\begin{aligned}
\bar{D}=\left\{1+\alpha \bar{\alpha}^{*}+\tau_{k}(\right. & -a_{11} x^{*}-k_{1}+a_{21} y^{*} \bar{\alpha}^{*}-a_{12} x^{*} \alpha \\
- & \left.\left.\left(a_{22} y^{*}+k_{2}\right) \alpha \bar{\alpha}^{*}\right) e^{-i \omega_{k} \tau_{k}}\right\}^{-1}
\end{aligned}
$$

such that $\left\langle q^{*}(s), q(\theta)\right\rangle=1,\left\langle q^{*}(s), \bar{q}(\theta)\right\rangle=0$.
Then when $\theta=0$, the system

$$
\dot{x}_{t}=A(\mu) x_{t}+R(\mu) x_{t}
$$

is equivalent to the system (29), where $x_{t}(\theta)=x(t+\theta), \theta \in$ $[-1,0]$. For $\psi \in \mathbb{C}^{1}\left([0,1],\left(\mathbb{R}^{2}\right)^{*}\right)$, define

$$
A^{*} \psi(s)= \begin{cases}-\frac{d \psi(s)}{d s}, & s \in(0,1], \\ \int_{-1}^{0} d \eta^{T}(t, 0) \psi(-t), & s=0,\end{cases}
$$

and a bilinear inner product

$\langle\psi(s), \phi(\theta)\rangle$

$$
=\bar{\psi}(0) \phi(0)-\int_{-1}^{0} \int_{\xi=0}^{\theta} \bar{\psi}(\xi-\theta) d \eta(\theta) \phi(\xi) d \xi,
$$

where $\eta(\theta)=\eta(\theta, 0)$, and let $A=A(0)$; then $A$ and $A^{*}$ are adjoint operators. By the discussion in Section 2, we know that $\pm i \omega_{k} \tau_{k}$ are eigenvalues of $A$. Thus, they are also eigenvalues of $A^{*}$. We first need to compute the eigenvector of $A$ and $A^{*}$ corresponding to $i \omega_{k} \tau_{k}$ and $-i \omega_{k} \tau_{k}$, respectively.

Suppose that $q(\theta)=(1, \alpha)^{T} e^{i \omega_{k} \tau_{k} \theta}$ is the eigenvector of $A$ corresponding to $i \omega_{k} \tau_{k}$. Then $A q(\theta)=i \omega_{k} \tau_{k} q(\theta)$. It follows from the definition of $A, L_{\mu} \phi$, and $\eta(\theta, \mu)$ that

In the following, we first compute the coordinates to describe the center manifold $C_{0}$ at $\mu=0$. Define

$$
z(t)=\left\langle q^{*}, x_{t}\right\rangle, \quad W(t, \theta)=x_{t}(\theta)-2 \operatorname{Re}\{z(t) q(\theta)\} .
$$

On the center manifold $C_{0}$, we have

$$
\begin{aligned}
W(t, \theta)= & W(z(t), \bar{z}(t), \theta) \\
= & W_{20}(\theta) \frac{z^{2}}{2}+W_{11}(\theta) z \bar{z}+W_{02}(\theta) \frac{\bar{z}^{2}}{2} \\
& +W_{30}(\theta) \frac{z^{3}}{6}+\cdots,
\end{aligned}
$$

where $z$ and $\bar{z}$ are local coordinates for center manifold $C_{0}$ in the direction of $q$ and $\bar{q}$. Note that $W$ is real if $x_{t}$ is real. We consider only real solutions. For the solution $x_{t} \in C_{0}$, since $\mu=0$, we have

$$
\dot{z}=i \omega_{k} \tau_{k} z+\left\langle q^{*}(\theta), f(0, W(z(t), \bar{z}(t), \theta)\right.
$$

$$
+2 \operatorname{Re}\{z(t) q(\theta)\})\rangle
$$




$$
\begin{aligned}
& =i \omega_{k} \tau_{k} z+\bar{q}^{*}(0) f(0, W(z(t), \bar{z}(t), 0) \\
& +2 \operatorname{Re}\{z(t) q(0)\}) \\
& \triangleq i \omega_{k} \tau_{k} z+\bar{q}^{*}(0) f_{0}(z, \bar{z})=i \omega_{k} \tau_{k} z+g(z, \bar{z}),
\end{aligned}
$$

where

$$
\begin{aligned}
g(z, \bar{z}) & =\bar{q}^{*}(0) f_{0}(z, \bar{z}) \\
& =g_{20}(\theta) \frac{z^{2}}{2}+g_{11}(\theta) z \bar{z}+g_{02}(\theta) \frac{\bar{z}^{2}}{2}+\cdots
\end{aligned}
$$

From (40) and (41), we have

$$
x_{t}(\theta)=\left(x_{1 t}(\theta), x_{2 t}(\theta)\right)^{T}=W(t, \theta)+z q(\theta)+\bar{z} \bar{q}(\theta) .
$$

In addition, $q(\theta)=(1, \alpha)^{T} e^{i \omega_{k} \tau_{k} \theta}$; then

$$
\begin{aligned}
x_{1 t}(0)= & z+\bar{z}+W_{20}^{(1)}(0) \frac{z^{2}}{2}+W_{11}^{(1)}(0) z \bar{z} \\
& +W_{02}^{(1)}(0) \frac{\bar{z}^{2}}{2}+O\left(|(z, \bar{z})|^{3}\right), \\
x_{2 t}(0)= & \alpha z+\bar{\alpha} \bar{z}+W_{20}^{(2)}(0) \frac{z^{2}}{2}+W_{11}^{(2)}(0) z \bar{z} \\
& +W_{02}^{(2)}(0) \frac{\bar{z}^{2}}{2}+O\left(|(z, \bar{z})|^{3}\right), \\
x_{1 t}(-1)= & z e^{-i \omega_{k} \tau_{k}}+\bar{z} e^{i \omega_{k} \tau_{k}}+W_{20}^{(1)}(-1) \frac{z^{2}}{2} \\
& +W_{11}^{(1)}(-1) z \bar{z}+W_{02}^{(1)}(-1) \frac{\bar{z}^{2}}{2}+O\left(|(z, \bar{z})|^{3}\right), \\
& +W_{11}^{(2)}(-1) z \bar{z}+W_{02}^{(2)}(-1) \frac{\bar{z}^{2}}{2}+O\left(|(z, \bar{z})|^{3}\right) . \\
x_{2 t}(-1)= & \alpha z e^{-i \omega_{k} \tau_{k}}+\bar{\alpha} \bar{z} e^{i \omega_{k} \tau_{k}}+W_{20}^{(2)}(-1) \frac{z^{2}}{2}
\end{aligned}
$$

By the definition of $f\left(\mu, x_{t}\right)$, we have

$$
g(z, \bar{z})
$$

$$
=\bar{D} \tau_{k}\left(1, \bar{\alpha}^{*}\right)\left[\begin{array}{c}
-a_{11} x_{1 t}(0) x_{1 t}(-1)-a_{12} x_{1 t}(0) x_{2 t}(-1) \\
a_{21} x_{2 t}(0) x_{1 t}(-1)-a_{22} x_{2 t}(0) x_{2 t}(-1)
\end{array}\right] .
$$

Substituting $x_{1 t}(0), x_{2 t}(0), x_{1 t}(-1)$, and $x_{2 t}(-1)$ into the above equation and comparing the coefficients with (43), we get

$$
\begin{gathered}
g_{20}=2 \bar{D} \tau_{k}\left(-a_{11}-a_{12} \alpha+a_{21} \alpha \bar{\alpha}^{*}-a_{22} \alpha^{2} \bar{\alpha}^{*}\right) e^{-i \omega_{k} \tau_{k}}, \\
g_{11}=2 \bar{D} \tau_{k}\left[\left(-a_{11}-a_{12} \bar{\alpha}+a_{21} \alpha \bar{\alpha}^{*}-a_{22} \alpha \bar{\alpha} \bar{\alpha}^{*}\right) e^{i \omega_{k} \tau_{k}}\right. \\
\left.-\left(a_{11}+a_{12} \alpha-a_{21} \bar{\alpha} \bar{\alpha}^{*}+a_{22} \alpha \bar{\alpha} \bar{\alpha}^{*}\right) e^{-i \omega_{k} \tau_{k}}\right] \\
g_{02}=2 \bar{D} \tau_{k}\left(-a_{11}-a_{12} \alpha+a_{21} \bar{\alpha} \bar{\alpha}^{*}-a_{22} \bar{\alpha}^{2} \bar{\alpha}^{*}\right) e^{i \omega_{k} \tau_{k}}, \\
g_{21} \bar{D} \tau_{k}\left[-a_{11}\left(2 W_{11}^{(1)}(-1)+W_{20}^{(1)}(-1)\right.\right. \\
\left.+W_{20}^{(1)}(0) e^{i \omega_{k} \tau_{k}}+2 W_{11}^{(1)}(0) e^{-i \omega_{k} \tau_{k}}\right) \\
+a_{12}\left(2 W_{11}^{(2)}(-1)+W_{20}^{(2)}(-1)\right. \\
\left.+\bar{\alpha} W_{20}^{(1)}(0) e^{i \omega_{k} \tau_{k}}+2 \alpha W_{11}^{(1)}(0) e^{-i \omega_{k} \tau_{k}}\right) \\
+a_{21} \bar{\alpha}^{*}\left(2 \alpha W_{11}^{(1)}(-1)+\bar{\alpha} W_{20}^{(1)}(-1)\right. \\
\left.+W_{20}^{(2)}(0) e^{i \omega_{k} \tau_{k}}+2 W_{11}^{(2)}(0) e^{-i \omega_{k} \tau_{k}}\right) \\
-a_{22} \bar{\alpha}^{*}\left(2 \alpha W_{11}^{(2)}(-1)+\bar{\alpha} W_{20}^{(2)}(-1)\right.
\end{gathered}
$$

In order to assure the value of $g_{21}$, we need to compute $W_{20}(\theta)$ and $W_{11}(\theta)$. From (34) and (40), we have

$$
\begin{aligned}
\dot{W} & =\dot{x}_{t}-\dot{z} q-\dot{\bar{z}} \bar{q} \\
& = \begin{cases}A W-2 \operatorname{Re}\left\{\bar{q}^{*}(0) f_{0} q(\theta)\right\}, & \theta \in[0,1) \\
A W-2 \operatorname{Re}\left\{\bar{q}^{*}(0) f_{0} q(\theta)\right\}+f_{0}, & \theta=0\end{cases} \\
& \triangleq A W+H(z, \bar{z}, \theta),
\end{aligned}
$$

where

$$
H(z, \bar{z}, \theta)=H_{20}(\theta) \frac{z^{2}}{2}+H_{11}(\theta) z \bar{z}+H_{02}(\theta) \frac{\bar{z}^{2}}{2}+\cdots
$$

Notice that near the origin on the center manifold $C_{0}$, we have

$$
\dot{W}=W_{z} \dot{z}+W_{\bar{z}} \dot{\bar{z}}
$$

thus, we have

$$
\begin{gathered}
\left(A-2 i \omega_{k} \tau_{k} I\right) W_{20}(\theta)=-H_{20}(\theta), \\
A W_{11}(\theta)=-H_{11}(\theta) .
\end{gathered}
$$


From (48), for $\theta \in[-1,0)$, we have

$$
\begin{aligned}
H & (z, \bar{z}, \theta) \\
& =-\bar{q}^{*}(0) f_{0} q(\theta)-q^{*}(0) \bar{f}_{0} \bar{q}(\theta)=-g q(\theta)-\bar{g} \bar{q}(\theta) .
\end{aligned}
$$

Comparing the coefficients with (48) gives that

$$
\begin{aligned}
& H_{20}(\theta)=-g_{20} q(\theta)-\bar{g}_{02} \bar{q}(\theta), \\
& H_{11}(\theta)=-g_{11} q(\theta)-\bar{g}_{11} \bar{q}(\theta) .
\end{aligned}
$$

From (51), (53), and the definition of $A$, we can get

$$
\dot{W}_{20}(\theta)=2 i \omega_{k} \tau_{k} W_{20}(\theta)+g_{20} q(\theta)+\bar{g}_{02} \bar{q}(\theta)
$$

Notice that $q(\theta)=q(0) e^{i \omega_{k} \tau_{k} \theta}$; we have

$W_{20}(\theta)=\frac{i g_{20}}{\omega_{k} \tau_{k}} q(0) e^{i \omega_{k} \tau_{k} \theta}+\frac{i \bar{g}_{02}}{3 \omega_{k} \tau_{k}} \bar{q}(0) e^{-i \omega_{k} \tau_{k} \theta}+E_{1} e^{2 i \omega_{k} \tau_{k} \theta}$,

where $E_{1}=\left(E_{1}^{(1)}, E_{1}^{(2)}\right) \in \mathbb{R}^{2}$ is a constant vector. In the same way, we can also obtain

$$
W_{11}(\theta)=-\frac{i g_{11}}{\omega_{k} \tau_{k}} q(0) e^{i \omega_{k} \tau_{k} \theta}+\frac{i \bar{g}_{11}}{\omega_{k} \tau_{k}} \bar{q}(0) e^{-i \omega_{k} \tau_{k} \theta}+E_{2}
$$

where $E_{2}=\left(E_{2}^{(1)}, E_{2}^{(2)}\right) \in \mathbb{R}^{2}$ is also a constant vector.

$$
B=\left[\begin{array}{cc}
2 i \omega_{k}-k_{1}+\left(a_{11}+k_{1}\right) x^{*} e^{-2 i \omega_{k} \tau_{k}} & a_{12} x^{*} e^{-2 i \omega_{k} \tau_{k}} \\
-a_{21} y^{*} e^{-2 i \omega_{k} \tau_{k}} & 2 i \omega_{k}-k_{2}+\left(a_{22} y^{*}+k_{2}\right) e^{-2 i \omega_{k} \tau_{k}}
\end{array}\right] .
$$

It follows that

$$
E_{1}=2 B^{-1}\left[\begin{array}{c}
-a_{11} e^{-i \omega_{k} \tau_{k}}-a_{12} \alpha e^{-i \omega_{k} \tau_{k}} \\
a_{21} \alpha e^{-i \omega_{k} \tau_{k}}-a_{22} \alpha^{2} e^{-i \omega_{k} \tau_{k}}
\end{array}\right]
$$

Similarly, substituting (56) and (60) into (58), we can get the formula of $E_{2}$, where
In what follows, we will compute $E_{1}$ and $E_{2}$. From the definition of $A$ and (51), we have

$$
\begin{gathered}
\int_{-1}^{0} d \eta(\theta) W_{20}(\theta)=2 i \omega_{k} \tau_{k} W_{20}(0)-H_{20}(0), \\
\int_{-1}^{0} d \eta(\theta) W_{11}(\theta)=-H_{11}(0) .
\end{gathered}
$$

From (48) and (49), we have

$$
\begin{aligned}
& H_{20}(0)=-g_{20} q(0)-\bar{g}_{02} \bar{q}(0) \\
& +2 \tau_{k}\left[\begin{array}{c}
-a_{11} e^{-i \omega_{k} \tau_{k}}-a_{12} \alpha e^{-i \omega_{k} \tau_{k}} \\
a_{21} \alpha e^{-i \omega_{k} \tau_{k}}-a_{22} \alpha^{2} e^{-i \omega_{k} \tau_{k}}
\end{array}\right], \\
& H_{11}(0)=-g_{11} q(0)-\bar{g}_{11} \bar{q}(0) \\
& +2 \tau_{k}\left[\begin{array}{l}
-a_{11} \operatorname{Re}\left\{e^{-i \omega_{k} \tau_{k}}\right\}-a_{12} \operatorname{Re}\left\{\alpha e^{-i \omega_{k} \tau_{k}}\right\} \\
a_{21} \operatorname{Re}\left\{\alpha e^{i \omega_{k} \tau_{k}}\right\}-a_{22} \operatorname{Re}\left\{\alpha \bar{\alpha} e^{-i \omega_{k} \tau_{k}}\right\}
\end{array}\right] .
\end{aligned}
$$

Substituting (55) and (59) into (57) and noticing that

$$
\begin{gathered}
{\left[i \omega_{k} \tau_{k} I-\int_{-1}^{0} e^{i \omega_{k} \tau_{k} \theta} d \eta(\theta)\right] q(0)=0,} \\
{\left[-i \omega_{k} \tau_{k} I-\int_{-1}^{0} e^{-i \omega_{k} \tau_{k} \theta} d \eta(\theta)\right] \bar{q}(0)=0,}
\end{gathered}
$$

we obtain

$$
\begin{aligned}
& {\left[2 i \omega_{k} \tau_{k} I-\int_{-1}^{0} e^{2 i \omega_{k} \tau_{k} \theta} d \eta(\theta)\right] E_{1}} \\
& \quad=2 \tau_{k}\left[\begin{array}{c}
-a_{11} e^{-i \omega_{k} \tau_{k}}-a_{12} \alpha e^{-i \omega_{k} \tau_{k}} \\
a_{21} \alpha e^{-i \omega_{k} \tau_{k}}-a_{22} \alpha^{2} e^{-i \omega_{k} \tau_{k}}
\end{array}\right],
\end{aligned}
$$

which leads to

$$
B E_{1}=2\left[\begin{array}{c}
-a_{11} e^{-i \omega_{k} \tau_{k}}-a_{12} \alpha e^{-i \omega_{k} \tau_{k}} \\
a_{21} \alpha e^{-i \omega_{k} \tau_{k}}-a_{22} \alpha^{2} e^{-i \omega_{k} \tau_{k}}
\end{array}\right],
$$

where

$$
\begin{aligned}
E_{2}= & 2\left[\begin{array}{cc}
a_{11} x^{*} & a_{12} x^{*} \\
-a_{21} y^{*} & a_{22} y^{*}
\end{array}\right]^{-1} \\
& \times\left[\begin{array}{l}
-a_{11} \operatorname{Re}\left\{e^{-i \omega_{k} \tau_{k}}\right\}-a_{12} \operatorname{Re}\left\{\alpha e^{-i \omega_{k} \tau_{k}}\right\} \\
a_{21} \operatorname{Re}\left\{\alpha e^{i \omega_{k} \tau_{k}}\right\}-a_{22} \operatorname{Re}\left\{\alpha \bar{\alpha} e^{-i \omega_{k} \tau_{k}}\right\}
\end{array}\right] .
\end{aligned}
$$

Thus, we can determine $W_{20}(\theta)$ and $W_{11}(\theta)$. Furthermore, we can determine each $g_{i j}$. Therefore, each $g_{i j}$ is determined 


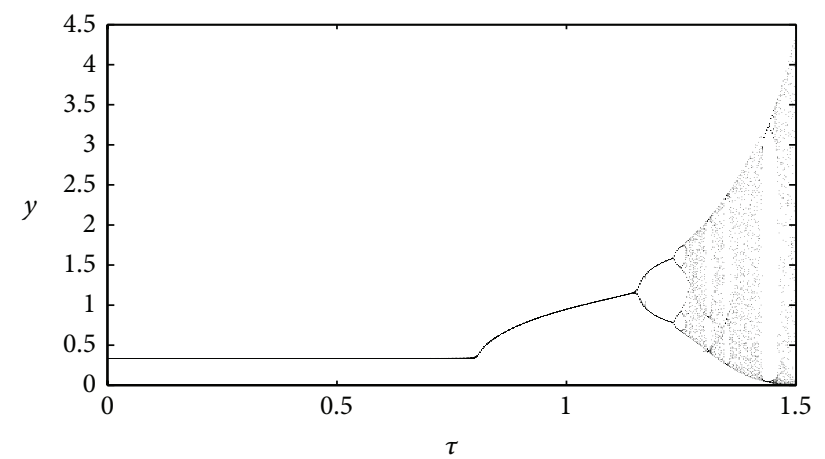

(a)

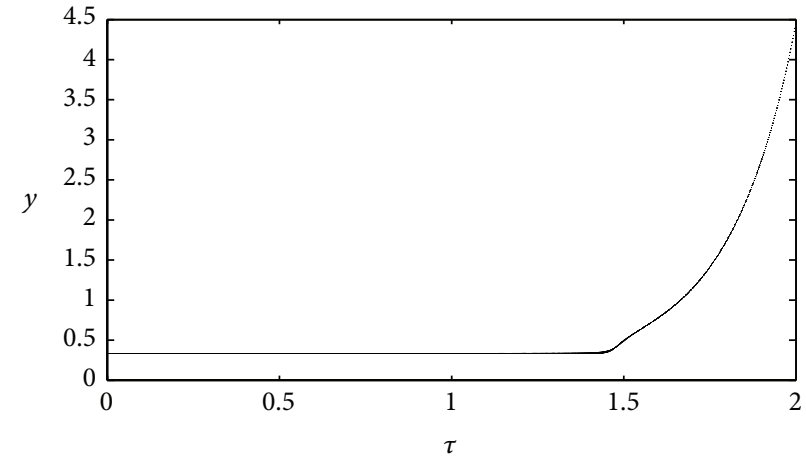

(b)

FIGURE 2: The bifurcation diagram of $y$ : (a) $k_{1}=k_{2}=0$; (b) $k_{1}=-0.2, k_{2}=0.5$.
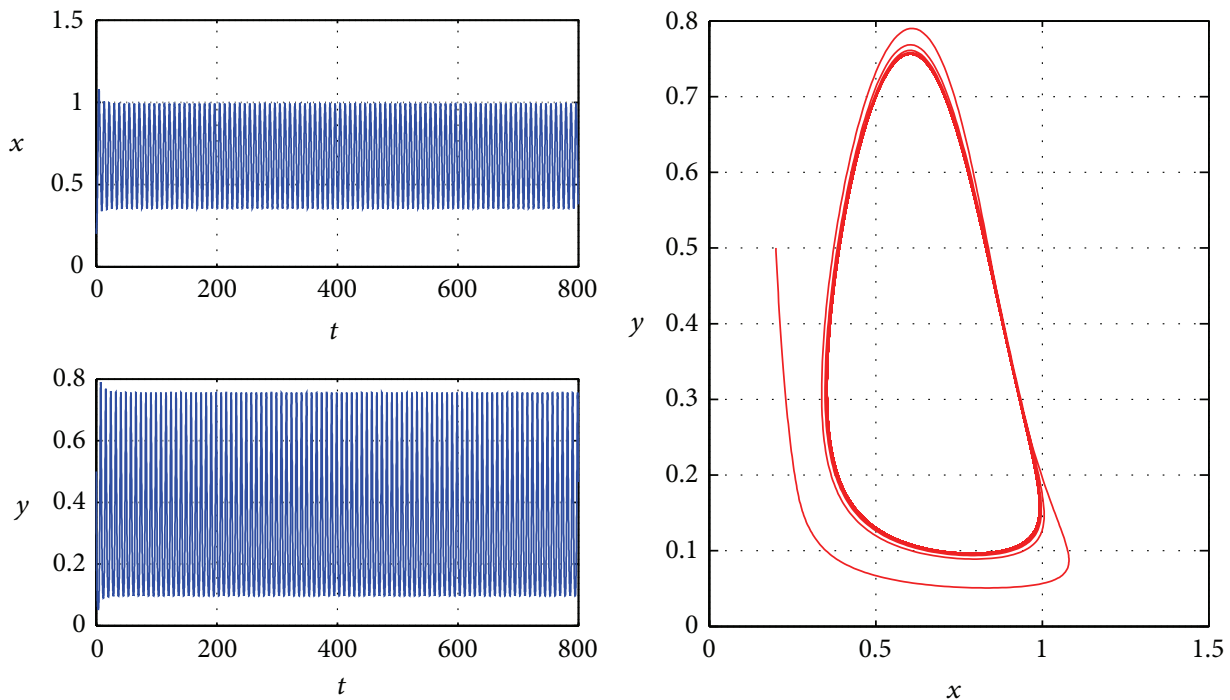

(a)
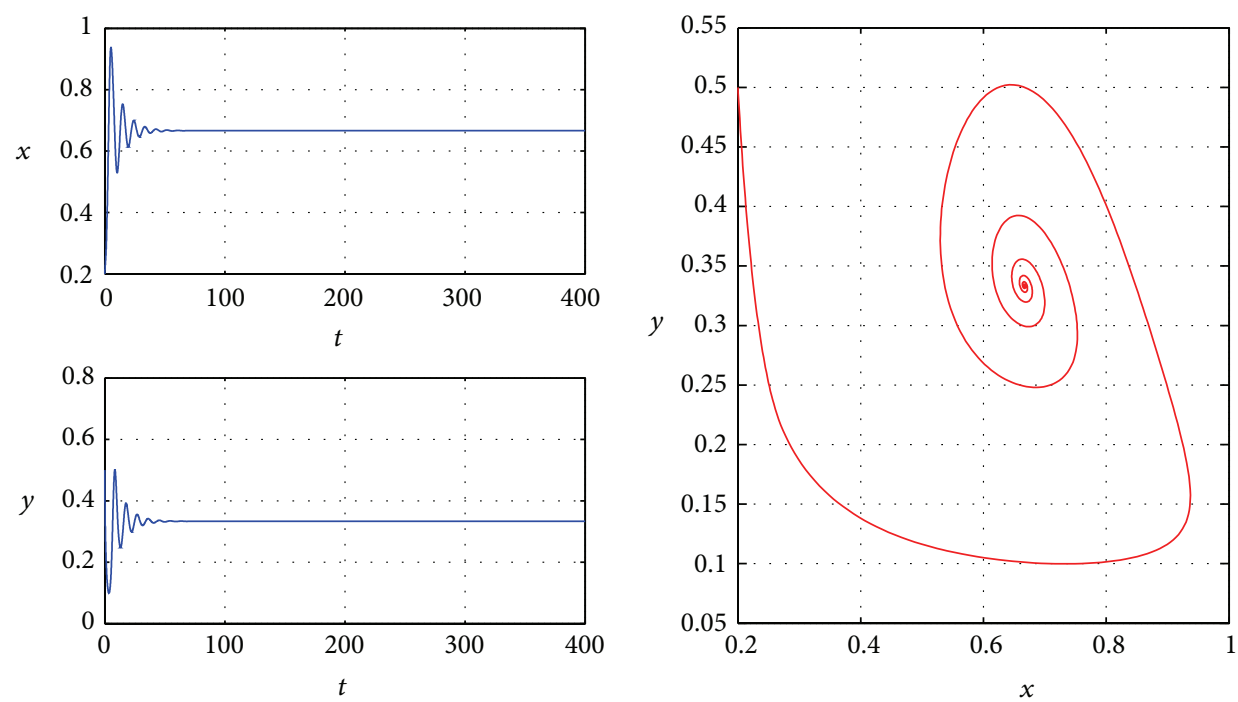

(b)

Figure 3: Waveform plot and phase plot of system (3) with $\tau=0.9$ : (a) $k_{1}=k_{2}=0$; (b) $k_{1}=-0.2, k_{2}=-0.5$. 

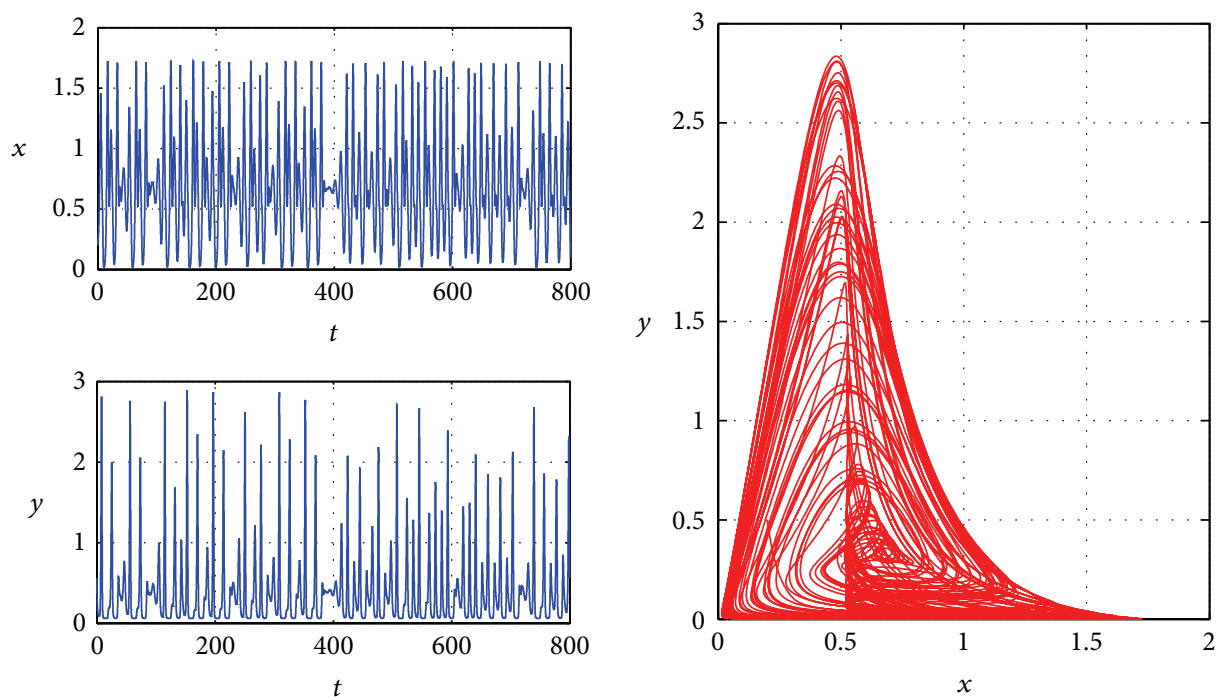

(a)
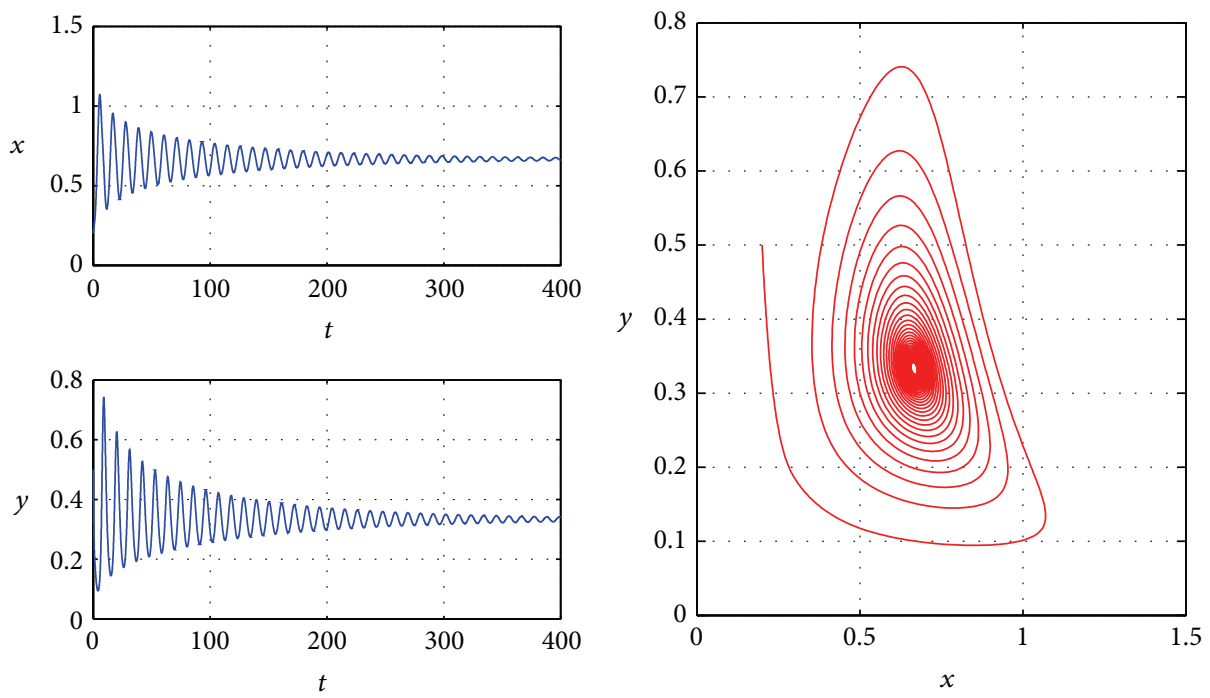

(b)

FIGURE 4: Waveform plot and phase plot of system (3) with $\tau=1.4$ : (a) $k_{1}=k_{2}=0$; (b) $k_{1}=-0.2, k_{2}=-0.5$.

by the parameters and delay in (5). Thus, we can compute the following values:

$$
\begin{aligned}
c_{1}(0) & =\frac{i}{2 \omega_{k} \tau_{k}}\left(g_{20} g_{11}-2\left|g_{11}\right|^{2}-\frac{1}{3}\left|g_{02}\right|^{2}\right)+\frac{1}{2} g_{21}, \\
\mu_{2} & =-\frac{\operatorname{Re}\left\{c_{1}(0)\right\}}{\operatorname{Re}\left\{\lambda^{\prime}(0)\right\}} \\
T_{2} & =-\frac{\operatorname{Im}\left\{c_{1}(0)\right\}+\mu_{2} \operatorname{Im}\left\{\lambda^{\prime}(0)\right\}}{\omega_{k} \tau_{k}}, \\
\beta_{2} & =2 \operatorname{Re}\left\{c_{1}(0)\right\}
\end{aligned}
$$

which determine the quantities of bifurcating periodic solutions in the center manifold at the critical value $\tau_{k}$; that is, $\mu_{2}$ determines the directions of the Hopf bifurcation: if $\mu_{2}>0(<0)$, then the Hopf bifurcation is supercritical (subcritical) and the bifurcation exists for $\tau>\tau_{0}\left(<\tau_{0}\right) ; \beta_{2}$ determines the stability of the bifurcation periodic solutions: the bifurcating periodic solutions are stable (unstable) if $\beta_{2}<$ 0 ( $>0$ ); and $T_{2}$ determines the period of the bifurcating periodic solutions: the period increases (decrease) if $T_{2}>$ $0(<0)$.

\section{Numerical Simulations}

In this section, we present numerical results to verify the analytical predictions obtained in the previous sections and use the delayed-feedback controller to control the Hopf bifurcation and chaos of system (3).

For the convenience of the calculation, we take the parameters of system (3) as $r_{1}=r_{2}=1, a_{11}=1, a_{12}=1$, 

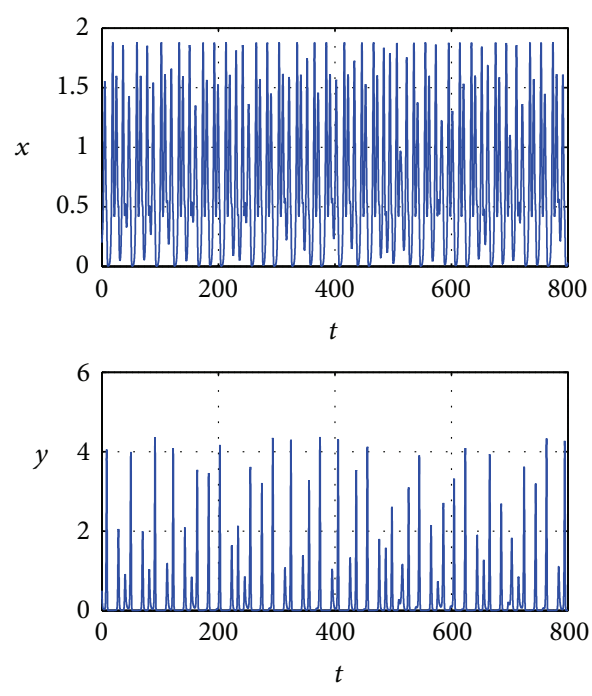

(a)
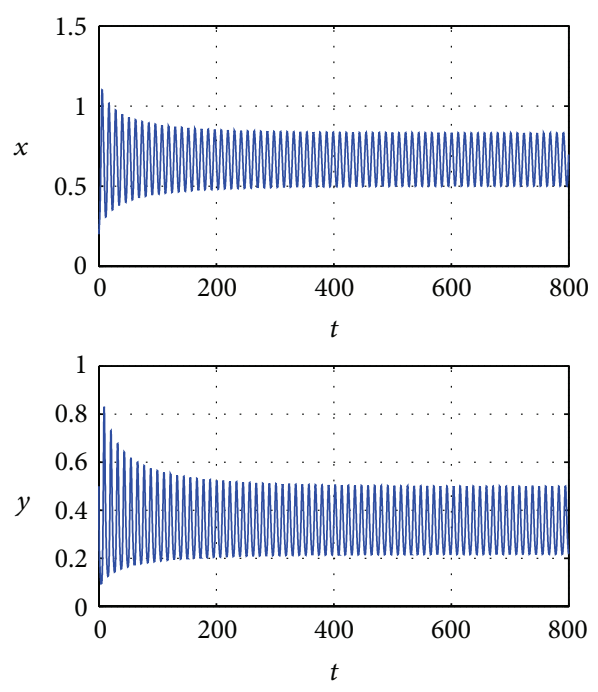

(b)
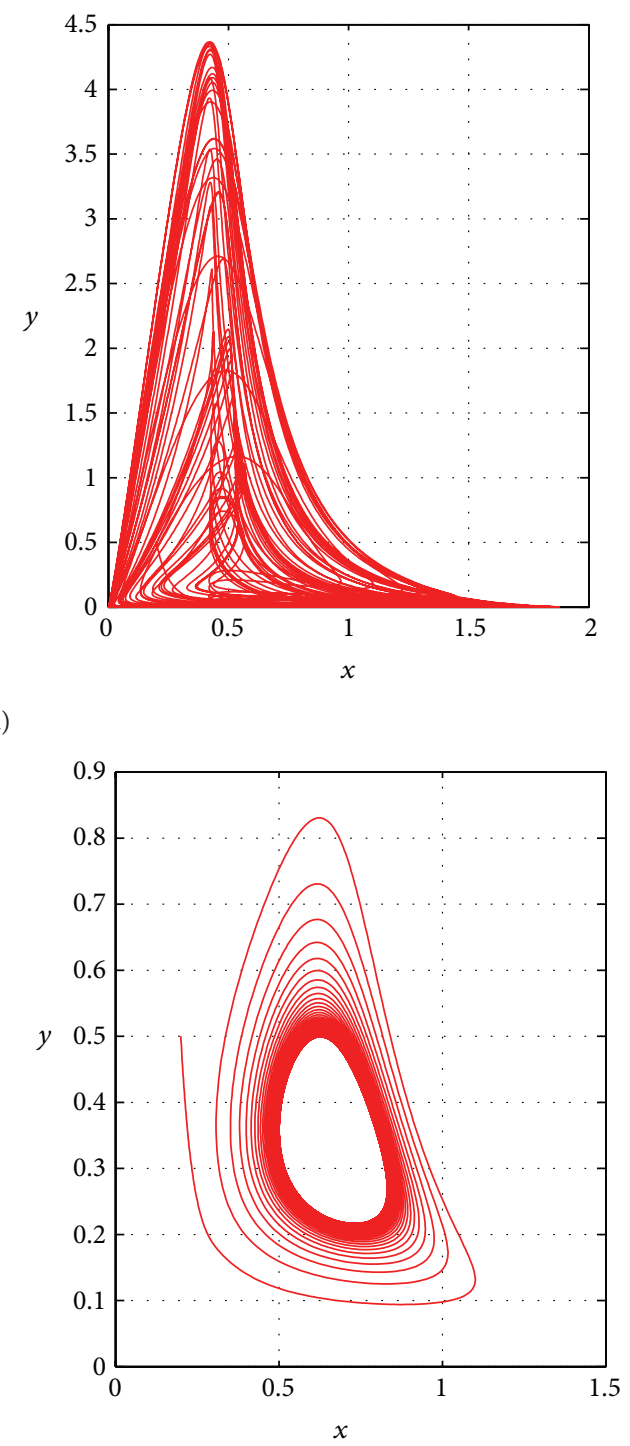

FigURE 5: Waveform plot and phase plot of system (3) with $\tau=1.5$ : (a) $k_{1}=k_{2}=0$; (b) $k_{1}=-0.2, k_{2}=-0.5$.

$a_{21}=2, a_{22}=1$. Then system (3) has a positive equilibrium $E_{*}=(2 / 3,1 / 3)$.

From [9], when $k_{1}=k_{2}=0$ or $\tau=0$, system (3) becomes system (2), which has a bifurcation point $\tau_{0}=$ 0.8072 , and when $\tau \in[0,0.8072), E_{*}$ is asymptotically stable and is unstable when $\tau>0.8072$, and with the increase of the delay $\tau$, chaos occurs via $t$ periodic-doubling bifurcation (Figure 2(a)), when $\tau=1.4, k_{1}=k_{2}=0$, system (3) becomes a chaotic one like illustrated by Figure 1.

Under the delayed-feedback control, if we choose $k_{1}=$ $-0.2, k_{2}=-0.5$, from the algorithm of Section 2, we can compute that $\tau_{0} \approx 1.4294$ and $\operatorname{Re}[d \lambda / d \tau]_{\tau=\tau_{0}}=0.1017>$ 0 ; hence from Theorem 2, when $\tau \in[0,1.4294), E_{*}$ is asymptotically stable and is unstable when $\tau>1.4294$, and when $\tau$ passes through the critical point $\tau_{0}$, a family of periodic orbits will bifurcate from $E_{*}$. Furthermore, from the algorithm of Section 3, we can compute that $g_{20}=1.4704+$ $2.4314 i, g_{11}=6.1433+8.6345 i, g_{02}=9.4686+15.7816 i$, $g_{21}=-24.6452+243.1853 i$; hence, based on the formula of the end of Section 3, we have $c_{1}(0)=-27.0460-64.6042 i$. It is easy to see that $\mu_{2}>0, \beta_{2}<0$. This means that the Hopf bifurcation is supercritical and the bifurcating periodic solutions are stable (Figure 5(b)).

By comparing the two bifurcation plots of Figure 2(a) and Figure 2(b), we observe that the bifurcation occurrence is postponed as the system is being controlled. Figure 3 shows that when $\tau=0.9$, if $k_{1}=k_{2}=0, E_{*}$ is unstable, and there are periodic orbits bifurcating from $E_{*}$ (Figure 3(a)); if we take $k_{1}=-0.2, k_{2}=-0.5, E_{*}$ becomes stable (Figure 3(b)). Figure 4 shows that when $\tau=1.4$, if $k_{1}=k_{2}=0, E_{*}$ is unstable, and system (3) becomes chaotic (Figure 4(a)); if we take $k_{1}=-0.2, k_{2}=-0.5$, then $E_{*}$ becomes stable 
and chaos vanishes (Figure 4(b)). Figure 5 shows that when $\tau=1.5$, if $k_{1}=k_{2}=0, E_{*}$ is unstable, and system (3) still exhibits chaotic behavior (Figure 5(a)); if we take $k_{1}=-0.2$, $k_{2}=-0.5$, then $E_{*}$ becomes unstable and stable periodic orbits bifurcate from $E_{*}$ (Figure 5(b)).

\section{Conclusion}

In this paper, we have studied a delayed Lotka-Volterra predator-prey system with time delayed feedback by using the theory of functional differential equation and Hassard's method. By analyzing the corresponding characteristic equations, the local stability of the positive equilibrium of system (3) was discussed.

We have obtained the estimated length of gestation delay which would not affect the stable coexistence of both prey and predator species at their equilibrium values. The existence of Hopf bifurcation for system (3) at the positive equilibrium was also established. From theoretical analysis it was shown that the larger values of gestation time delay cause fluctuation in individual population density and hence the system becomes unstable.

As the estimated length of delay to preserve stability and the critical length of time delay for Hopf-bifurcation are dependent upon the system parameters, it is possible to impose some control, which will prevent the possible abnormal oscillation in population density. Our results show that if we choose some appropriate parameters, the oscillation can be controlled to a stable equilibrium or a stable periodic orbit; that is to say, we can achieve the ecological equilibrium by adjusting the capture (or release) level.

\section{Conflict of Interests}

The authors declare that there is no conflict of interests regarding the publication of this paper.

\section{Acknowledgments}

This research is supported by Science and Technology Department of Henan Province (no.122300410417), Education Department of Henan Province (no.13A110108), 2013 Narure Science Foundation of Ningxia (no. NZ13096), 2013 Higher educational scientific research project of Ningxia (no. NGY2013086).

\section{References}

[1] J. Zhen and Z. Ma, "Stability for a competitive Lotka-Volterra system with delays," Nonlinear Analysis. Theory, Methods \& Applications, vol. 51, no. 7, pp. 1131-1142, 2002.

[2] Y. Saito, "The necessary and sufficient condition for global stability of a Lotka-Volterra cooperative or competition system with delays," Journal of Mathematical Analysis and Applications, vol. 268, no. 1, pp. 109-124, 2002.

[3] K. Gopalsamy and X.-Z. He, "Oscillations and convergence in an almost periodic competition system," Acta Applicandae Mathematicae, vol. 46, no. 3, pp. 247-266, 1997.
[4] X. H. Tang and X. Zou, "Global attractivity of non-autonomous Lotka-Volterra competition system without instantaneous negative feedback," Journal of Differential Equations, vol. 192, no. 2, pp. 502-535, 2003.

[5] Y. Song and Y. Peng, "Stability and bifurcation analysis on a logistic model with discrete and distributed delays," Applied Mathematics and Computation, vol. 181, no. 2, pp. 1745-1757, 2006.

[6] H. Zhao and Y. Lin, "Hopf bifurcation in a partial dependent predator-prey system with delay," Chaos, Solitons and Fractals, vol. 42, no. 2, pp. 896-900, 2009.

[7] Y. Song, Y. Peng, and J. Wei, "Bifurcations for a predator-prey system with two delays," Journal of Mathematical Analysis and Applications, vol. 337, no. 1, pp. 466-479, 2008.

[8] X.-P. Yan, "Stability and Hopf bifurcation for a delayed preypredator system with diffusion effects," Applied Mathematics and Computation, vol. 192, no. 2, pp. 552-566, 2007.

[9] X.-P. Yan and C.-H. Zhang, "Hopf bifurcation in a delayed Lokta-Volterra predator-prey system," Nonlinear Analysis. Real World Applications, vol. 9, no. 1, pp. 114-127, 2008.

[10] J. Zhang, Z. Jin, J. Yan, and G. Sun, "Stability and Hopf bifurcation in a delayed competition system," Nonlinear Analysis. Theory, Methods \& Applications, vol. 70, no. 2, pp. 658-670, 2009.

[11] H. Chen, "Chaos control and global synchronization of Liu chaotic systems using linear balanced feedback control," Chaos, Solitons and Fractals, vol. 40, no. 1, pp. 466-473, 2009.

[12] W. Chen, "Dynamics and control of a financial system with time-delayed feedbacks," Chaos, Solitons and Fractals, vol. 37, pp. 1198-1207, 2008.

[13] D. Ghosh, A. R. Chowdhury, and P. Saha, "Bifurcation continuation, chaos and chaos control in nonlinear Bloch system," Communications in Nonlinear Science and Numerical Simulation, vol. 13, no. 8, pp. 1461-1471, 2008.

[14] A. El-Gohary and A. S. Al-Ruzaiza, "Chaos and adaptive control in two prey, one predator system with nonlinear feedback," Chaos, Solitons and Fractals, vol. 34, no. 2, pp. 443-453, 2007.

[15] J. Lü and S. Zhang, "Controlling Chen's chaotic attractor using backstepping design based on parameters identification," Physics Letters A, vol. 286, pp. 148-152, 2001.

[16] S. Boccaletti, C. Grebogi, Y.-C. Lai, H. Mancini, and D. Maza, "The control of chaos: theory and applications," Physics Reports, vol. 329, no. 3, pp. 103-197, 2000.

[17] Y. Song and J. Wei, "Bifurcation analysis for Chen's system with delayed feedback and its application to control of chaos," Chaos, Solitons \& Fractals, vol. 22, no. 1, pp. 75-91, 2004.

[18] S. Ruan and J. Wei, "On the zeros of transcendental functions with applications to stability of delay differential equations with two delays," Dynamics of Continuous, Discrete \& Impulsive Systems. A. Mathematical Analysis, vol. 10, no. 6, pp. 863-874, 2003.

[19] J. K. Hale and S. M. Verduyn Lunel, Introduction to FunctionalDifferential Equations, vol. 99 of Applied Mathematical Sciences, Springer, New York, NY, USA, 1993.

[20] B. D. Hassard, N. D. Kazarinoff, and Y. H. Wan, Theory and Applications of Hopf Bifurcation, vol. 41 of London Mathematical Society Lecture Note Series, Cambridge University Press, New York, NY, USA, 1981. 


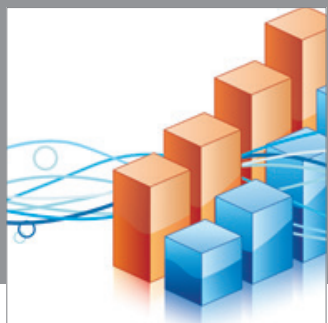

Advances in

Operations Research

mansans

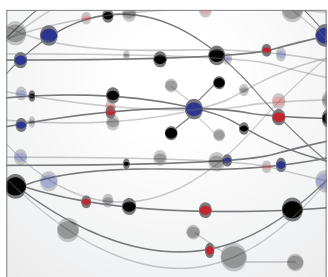

The Scientific World Journal
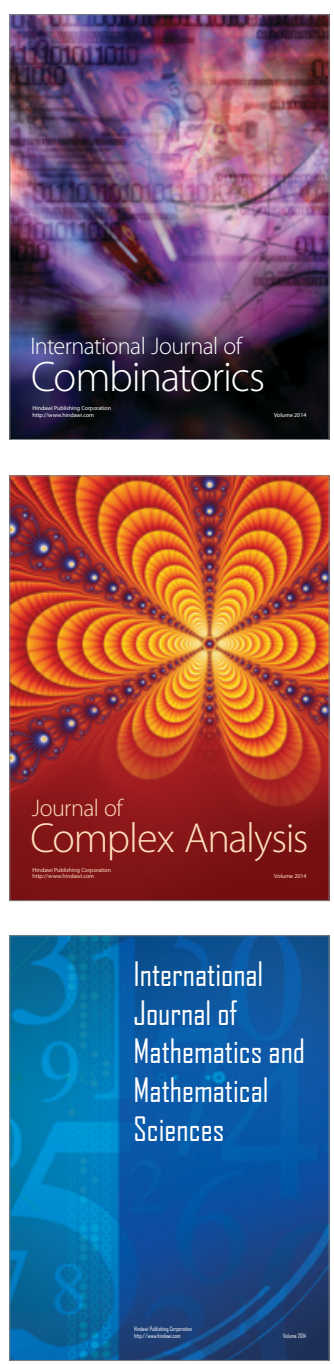
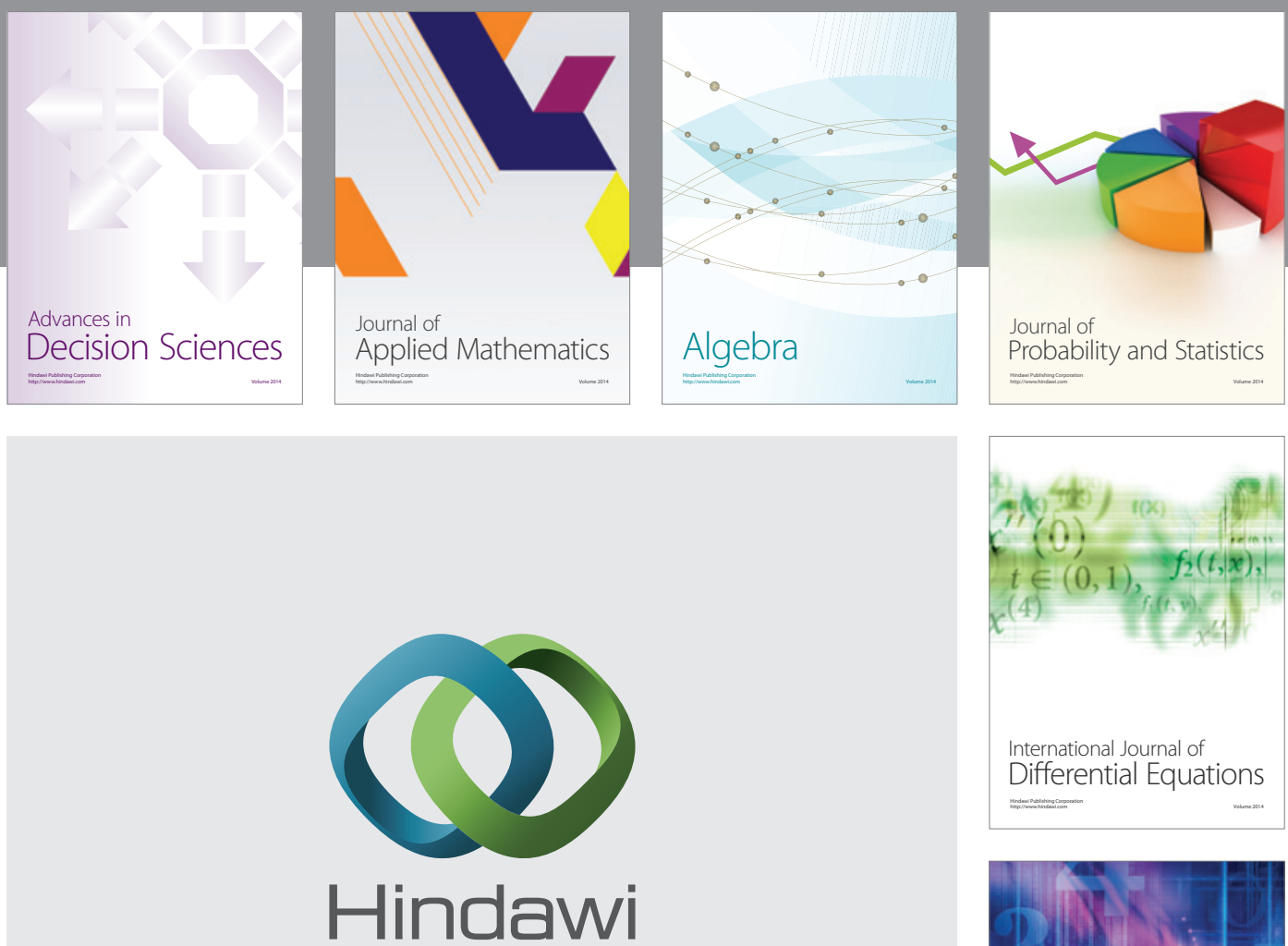

Submit your manuscripts at http://www.hindawi.com
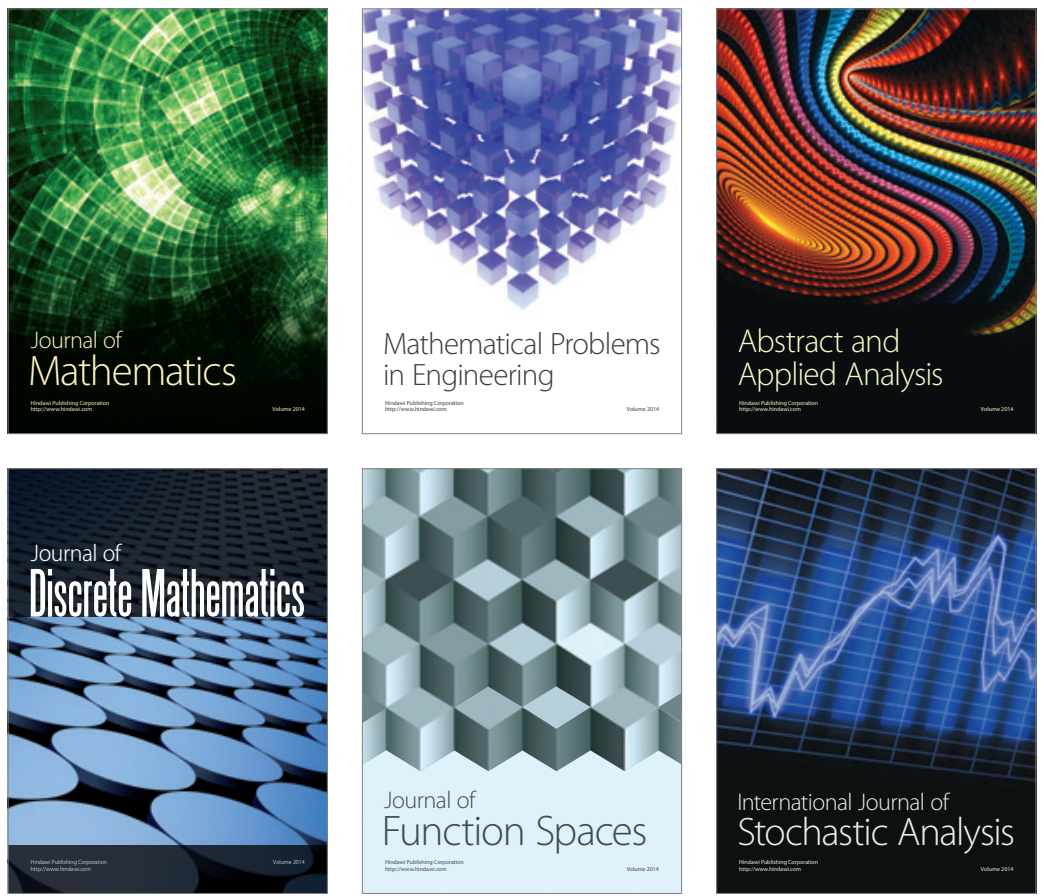

Journal of

Function Spaces

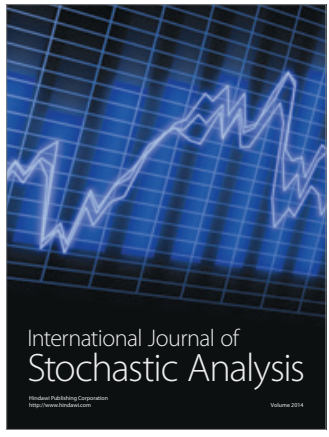

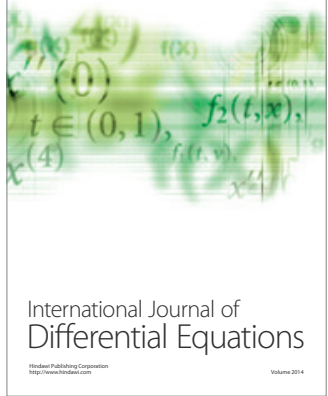
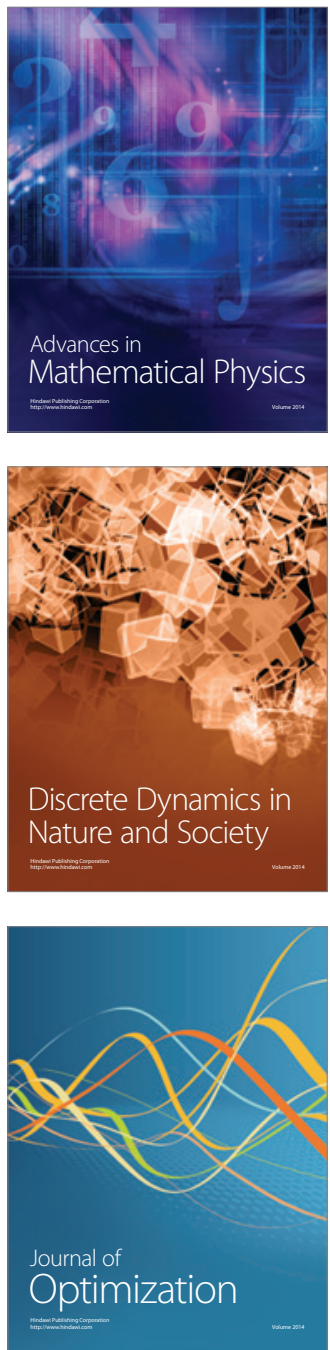